\title{
Invasive Intestinal Aspergillosis in the Setting of Autologous Graft Versus Host Disease after Hematopoietic Cell Transplant for Multiple Myeloma

\author{
John S Wang ${ }^{1}$, Omar Elghawy ${ }^{1}$, Rachel M Whitehair ${ }^{2}$, Tamila L Kindwall-Keller ${ }^{*}$ \\ ${ }^{1}$ School of Medicine, University of Virginia, Charlottesville, VA, USA \\ ${ }^{2}$ University of Virginia, Department of Pathology, Charlottesville, VA, USA \\ ${ }^{3}$ University of Virginia, Division of Hematology and Oncology, Charlottesville, VA, USA
}

\begin{abstract}
Autologous stem-cell transplant is used to treat a variety of neoplastic syndromes such as multiple myeloma, chronic myeloid leukemia, and non-Hodgkin's lymphoma. In spite of its efficacy compared to other treatments, it predisposes patients to opportunistic infections and in rare cases, autologous graft versus host disease. In recent years, one opportunistic infection in particular, invasive aspergillosis, has become increasingly more common although survival rates remain low due to difficulty in diagnosis. While relatively benign in the immunocompetent, aspergillosis can be deadly in the immunocompromised. Herein, we present the case of a 68-year-old male with invasive intestinal aspergillosis secondary to autologous graft versus host disease. This case highlights the importance of evaluating immunocompromised patients for aspergillosis when persistent symptoms return otherwise negative bacterial and fungal cultures. In addition, this case reinforces a trend in the literature supporting an association between intestinal graft versus host disease and intestinal aspergillosis which has important implications for guiding anti-mold prophylaxis in this patient population.
\end{abstract}

Keywords

Aspergillus, Autologous hematopoietic cell transplant, Graft versus host disease

\section{Introduction}

In recentyears, progress has been made in the development of new treatments for multiple myeloma increasing disease response and overall survival [1]. Autologous hematopoietic cell transplant (AHCT) is the standard of care for fit patients younger than age 71 [2]. However, despite the success of treatment, AHCT is associated with significant morbidity including chemotherapy toxicity, opportunistic infections, relapse, secondary malignancy and rarely, autologous graft versus host disease (GVHD) [3]. Autologous GVHD has been associated with widespread damage to various tissues including skin, gastrointestinal tract, lung, and liver [4-5]. Although the pathophysiology of the condition is not well understood, it is believed to occur when either autoreactive or microchimeric T cells attack the host tissue [6]. GVHD can predispose patients to opportunistic infections through immune dysregulation, requiring treatment with immunosuppression, thereby increasing the risk of infection [7].

One of the most deadly and difficult to treat opportunistic fungal infections in hematopoietic cell transplant (HCT) is aspergillus [8]. Aspergillus is commonly found in soil and decaying vegetables and causes disease through inhalation of its airborne spores or ingestion of its carcinogenic aflatoxin [9]. It presents mildly in most patients, but can cause severe complications in individuals who are immunocompromised [10]. Invasive aspergillosis can be a fatal systemic infection due to spread from the lungs to the central nervous system (CNS), heart, kidneys, and gastrointestinal tract $[11,12]$. While aspergillosis has become more prominent in recent years, especially in chemotherapy and HCT recipients, it is still relatively rare compared to other opportunistic fungi such as Candida [13]. This is especially true in the setting of

*Corresponding author: Tamila L Kindwall-Keller, University of Virginia, Division of Hematology and Oncology, Charlottesville, PO Box 800716, VA, 22908, USA, Fax: 434-243-0064, Tel: 434297-5026, E-mail: TLK5DE@hscmail.mcc.virginia.edu

Accepted: July 26, 2021

Published online: July 28, 2021

Citation: Wang JS, Elghawy O, Whitehair RM, et al. (2021) Invasive Intestinal Aspergillosis in the Setting of Autologous Graft Versus Host Disease after Hematopoietic Cell Transplant for Multiple Myeloma. J Clin Surg Pathol 3(1):27-30 
Citation: Wang JS, Elghawy O, Whitehair RM, et al. (2021) Invasive Intestinal Aspergillosis in the Setting of Autologous Graft Versus Host Disease after Hematopoietic Cell Transplant for Multiple Myeloma. J Clin Surg Pathol 3(1):27-30

AHSCT which is less immunosuppressive than allogeneic HCT [14]. Patients rarely develop autologous GVHD predisposing them to opportunistic infections [4]. Herein, we present a patient with invasive intestinal aspergillosis in the setting of autologous GVHD after AHCT for multiple myeloma.

\section{Case Presentation}

A 68-year-old male with multiple myeloma was referred for evaluation for AHCT. At the time of diagnosis, he presented with worsening fatigue, increased swelling in the anterior chest wall and sternal chest pain. He had hypercalcemia, anemia (hemoglobin $6.4 \mathrm{~g} / \mathrm{dL}$ ) and renal failure (creatinine 4.4 $\mathrm{mg} / \mathrm{dL}$ ). Serum protein electrophoresis with immunofixation identified an IgG Lambda monoclonal protein. Lambda free light chains were 13,120 with a free light chain ratio of 0.15 . Skeletal survey revealed innumerable lytic lesions throughout the bony calvarium. A soft tissue mass with its epicenter in the anterior mediastinum appeared to invade the body wall just left of midline by CT imaging. Biopsy of a pelvic mass revealed a lymphoplasmacytic process favoring a plasmacytoma, positive for Congo Red staining. Cytogenetic/FISH analysis revealed that $90 \%$ of the metaphase cells showed rearrangement of chromosome 1, monosomy of chromosomes 13, 14 and 17 and partial gain of chromosome 15.

At consultation for AHCT, the patient had a Karnofsky performance status of $60 \%$. Examination revealed diffuse nontender swelling of the anterior chest wall. Laboratory analysis revealed a calcium 7.2, hemoglobin $10.6 \mathrm{~g} / \mathrm{dL}$ and creatinine of $1.5 \mathrm{mg} / \mathrm{dL}$. His kappa light chain was $<0.54$ and lambda light chain was 3.53. He had responded well to induction chemotherapy with a very good partial remission. Bone marrow aspirate and biopsy revealed no increase in plasma cells. Immunohistochemistry and flow cytometry for CD138 confirmed that the plasma cell population was not increased. Melphalan $200 \mathrm{mg} / \mathrm{m}^{2}$ (total dose $380 \mathrm{mg}$ ) conditioning was given two days prior to the AHCT. Institutional standard viral and fungal prophylaxis with acyclovir and fluconazole were given. Neutrophil and platelet engraftment occurred on day +11 and day +13 , respectively.

An abdomen/pelvic CT scan performed 8 days after AHCT to evaluate ongoing neutropenic fever, diarrhea and cramping revealed severe diffuse wall thickening of the sigmoid colon, distal descending colon and distal transverse colon. Since the CT scan results were concerning for infectious colitis, broadspectrum antibiotics were started. Exhaustive workup for an infectious etiology was unrevealing: Clostridium difficile was negative and stool studies were negative for Campylobacter jejuni, cryptosporidium species, Giardia, microsporidia species, Cyclospora species, acid fast bacilli, cytomegalovirus, histoplasmosis, cryptococcus and norovirus. A repeat CT was performed 5 days after the initial CT scan to evaluate tenderness on abdominal palpation showed moderate bowel wall thickening, with some residual abnormal mucosal thickening within the sigmoid and descending colon, consistent with improving colitis. Flexible sigmoidoscopy and biopsies were performed to evaluate the diarrhea despite improvement on imaging. Visualization of the sigmoid colon revealed erythematous, ulcerated mucosa consistent with infectious colitis. Biopsies were consistent with acute colitis.

Nineteen days after AHCT, due to worsening gastrointestinal symptoms, a repeat CT abdomen/pelvis was performed showing interval development of diffuse bowel wall edema and hyperenhancement of the intestinal mucosa from the duodenum through the rectum. Differential diagnosis included shock bowel, autologous GVHD, severe infectious or inflammatory etiologies. Methylprednisolone and octreotide were started for the treatment of autologous GVHD associated diarrhea. Cyclosporine was added when the patient's symptoms failed to improve on methylprednisolone at $2 \mathrm{mg} / \mathrm{kg}$. Given concerns that the autologous intestinal GVHD was not improving with immunosuppression despite improvement on recent CT imaging, the diarrhea was reevaluated by a second flexible sigmoidoscopy. Visualization of the sigmoid colon revealed a moderately erythematous, congested mucosa with scattered superficial erosions and granulation tissue throughout (Figure 1A). Biopsies were consistent with severe autologous intestinal GVHD (Figures $1 \mathrm{~B}$ and Figure $1 \mathrm{C})$.

Although treatment was initially successful in reducing the gastrointestinal symptoms, the diarrhea became bloody with as many as eight episodes per day. Repeat endoscopy revealed blood in the entire gastrointestinal tract, and tissue friability in the recto-sigmoid colon. CT scan showed worsening gastrointestinal inflammation despite treatment with methylprednisolone, cyclosporine, mycophenolate mofetil, and etanercept during the hospitalization. The patient was made comfort care and died shortly after.

An autopsy was requested to determine the source of the gastrointestinal bleeding and cause of death. The entire gastrointestinal tract was filled with blood. Esophagus was remarkable for mid-esophageal elongated ulcerations located approximately $11 \mathrm{~cm}$ from the gastroesophageal junction. Stomach and small intestine showed serosal petechiae throughout the entire length of both. Small intestine, stomach and pancreas showed marked autolysis. Large intestine showed multiple diverticula and dilated crypts with karyorrhectic debris. Methenamine silver stain demonstrated the presence of septate hyphae, branching in acute angles, invading the epithelium and submucosa (Figure 2). Autopsy report revealed the final cause of death to be an invasive intestinal aspergillosis secondary to immunosuppression for autologous GVHD after AHCT for multiple myeloma.

\section{Discussion}

While GVHD is a common complication of allogeneic HCT, it is rare in AHCT [6]. In this report, our patient's AHCT was complicated by autologous intestinal GVHD. Autologous intestinal GVHD should be considered in the event of gastrointestinal symptoms with negative viral, bacterial, and fungal cultures [15]. The few cases documented in the literature are consistent with our patient's presentation: severe diarrhea, enterocolitis, colonic ulcers, and abdominal pain with an intestinal biopsy confirming apoptotic crypts and abnormal mucosa [15] Treatment for autologous GVHD requires a regimen of immunosuppression to control 
Citation: Wang JS, Elghawy O, Whitehair RM, et al. (2021) Invasive Intestinal Aspergillosis in the Setting of Autologous Graft Versus Host Disease after Hematopoietic Cell Transplant for Multiple Myeloma. J Clin Surg Pathol 3(1):27-30
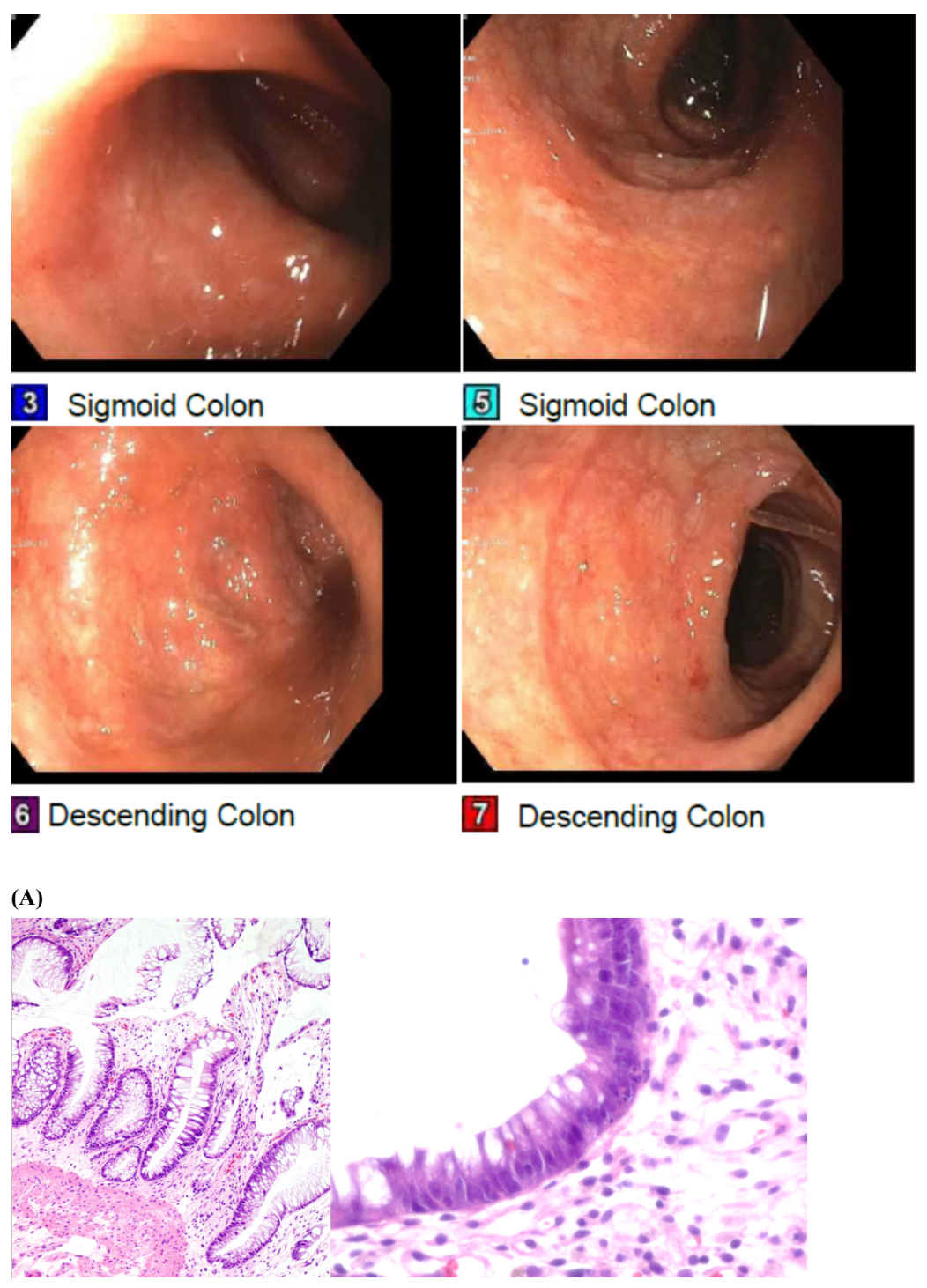

(B)

(C)

Figure 1: A) Flexible sigmoidoscopy revealing erythematous mucosa as well as multiple erosions and bleeding throughout the length of the recto-sigmoid colon; B) Colonic glands with apoptosis at the crypt bases consistent with GVHD (20x H\&E) and C) A higher magnification of apoptotic cells at a crypt base (40x H\&E).

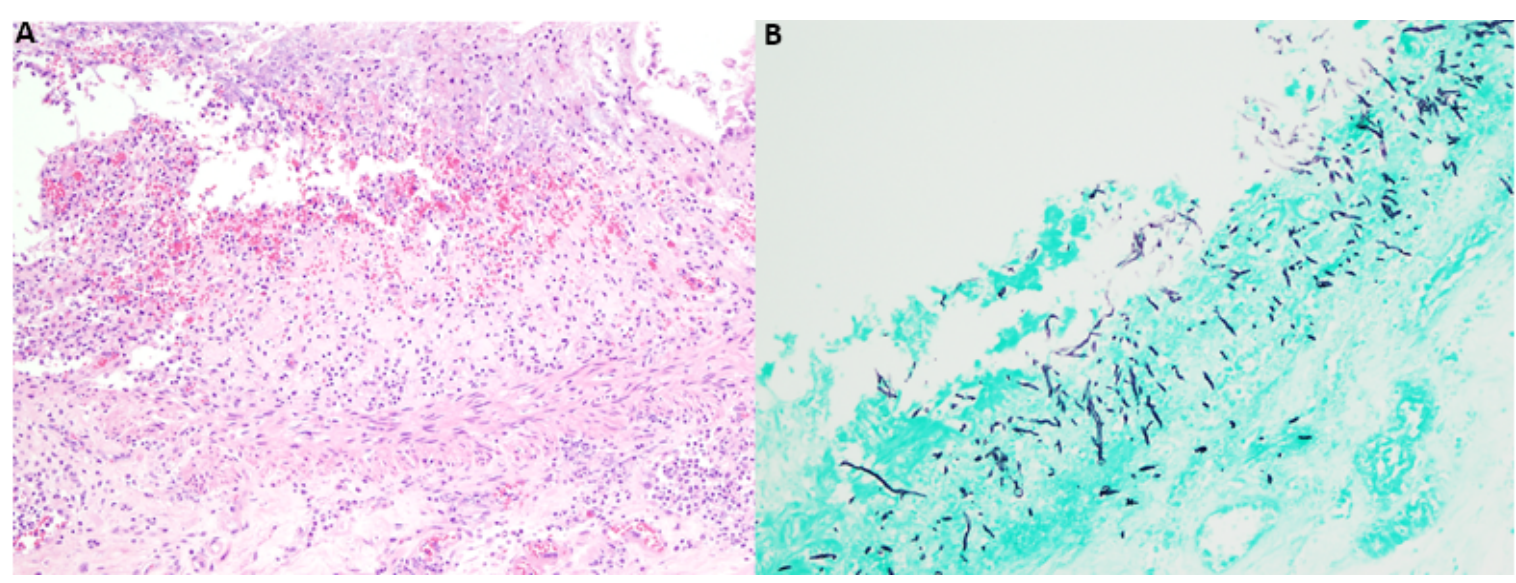

Figure 2: A) A section of large bowel at the time of autopsy with complete loss of glands and replacement by granulation tissue (10x, $\mathrm{H} \& \mathrm{E})$; B) A Grocott Methenamine Silver stain performed on the autopsy bowel specimen, highlights numerous fungal hyphae. Some of the hyphae demonstrate 45 degree angle branching and are invading into the submucosa. These features are suggestive of an invasive aspergillus infection (20x GMS). 
Citation: Wang JS, Elghawy O, Whitehair RM, et al. (2021) Invasive Intestinal Aspergillosis in the Setting of Autologous Graft Versus Host Disease after Hematopoietic Cell Transplant for Multiple Myeloma. J Clin Surg Pathol 3(1):27-30

the disease [16]. Intestinal GVHD often resolves gradually after treatment with steroids - in this case, the patient received a regimen of methylprednisolone, cyclosporine, mycophenolate mofetil, and etanercept without improvement [16]. The immunosuppression predisposed this patient to an opportunistic fungal infection, invasive aspergillosis.

Aspergillosis in the immunocompromised encompasses infections of multiple organ systems as it can disseminate through both tissue invasion and hematogenously [11]. Invasive aspergillosis is very rarely seen in immunocompetent hosts as they typically have sufficient levels of alveolar macrophages to phagocytose the fungal conidia and hyphae before infection disseminates [17]. However, the frequency of invasive aspergillosis has increased in immunocompromised patients due to the increase in number of HCT and solid-organ transplants performed every year [11]. Since aspergillus is most commonly transmitted through inhalation of its spores, pulmonary infections constitute more than $90 \%$ of invasive Aspergillus cases [17]. In these patients, the subsequent lung cavitation and chronic necrosis are often fatal [17]. Aspergillus has been observed to seed to the heart, skin, CNS, and gastrointestinal tract via hematogenous spread in extrapulmonary cases [11]. Our case report demonstrates a rare example of isolated aspergillosis of the gastrointestinal tract which has only been documented in the clinical literature a few times prior [18].

The differential diagnosis for patients experiencing gastrointestinal complications after AHCT is expansive and includes pseudomembranous enterocolitis, typhlitis, viral enteritis, and benign pneumatosis intestinalis [19] Early recognition of the symptoms of GVHD with confirmational biopsy can help determine the course of clinical treatment. Despite a low overall risk of invasive aspergillosis in AHCT, studies have shown as high as a $27 \%$ risk of invasive aspergillosis in the subgroup of patients with intestinal GVHD in allogeneic HCT suggesting an association between the two [13]. Careful selection of antifungal prophylaxis is especially relevant in this situation as aspergillus is resistant to fluconazole prophylaxis given for the more common Candida albicans [20]. Therefore, as demonstrated in this case, making the association between invasive aspergillus and intestinal GVHD is pivotal in the management of these at-risk populations.

\section{Acknowledgments}

We would like to thank the Pathology, Gastroenterology, and Hematology and Oncology departments of the University of Virginia Medical Center for their technical assistance in the workup of this case.

\section{Conflict of Interest}

The authors have no financial interest or any conflict of interest relevant to the content of this article.

\section{References}

1. Kumar SK, Callander NS, Hillengass J, et al. (2019) NCCN guidelines insights: Multiple myeloma, version 1.2020. J Natl Compr Canc Netw 17: 1154-1165.
2. Moreau P, Touzeau C, Vij R, et al. (2020) Newly diagnosed myeloma in 2020. Am Soc Clin Oncol Educ Book 40: 1-15.

3. Majhail NS (2017) Long Term complications after hematopoietic cell transplantation. Hematol Oncol Stem Cell Ther 10: 220-227.

4. Sloane JP, Powles RL (1980) Graft-versus-host disease in recipients of syngeneic bone marrow. Lancet 1: 253-254.

5. Fidler C, Klumpp T, Mangan K, et al. (2012) Spontaneous graft versus host disease occurring in a patient with multiple myeloma after autologous stem cell transplant. Am J Hematol 87: 219-221.

6. Holmberg L, Kikuchi K, Gooley TA, et al. (2006) Gastrointestinal graft-versus-host disease in recipients of autologous hematopoietic stem cells: Incidence, risk factors, and outcome. Biol Blood Marrow Transplant 12: 226-234.

7. Miller HK, Braun TM, Stillwell T, et al. (2017) Infectious risk after allogeneic hematopoietic cell transplant complicated by acute graftversus-host disease. Biol Blood Marrow Transplant 23: 522-528.

8. Kosmidis C, Denning DW (2015) The clinical spectrum of pulmonary aspergillosis. Thorax 70: 270-277.

9. Paulussen C, Hallsworth JE, Álvarez-Pérez S, et al. (2017) Ecology of aspergillosis: insights into the pathogenic potency of Aspergillus fumigatus and some other Aspergillus species. Microb Biotechnol 10: 296-322.

10. Walsh TJ, Anaissie EJ, Denning DW, et al. (2008) Treatment of aspergillosis: Clinical practice guidelines of the infectious diseases society of America. Clin Infect Dis 46: 327-360.

11. Gregg KS, Kauffman CA (2015) Invasive aspergillosis: Epidemiology, clinical aspects, and treatment. Semin Respir Crit Care Med 36: 662-672.

12. Panackal AA, Bennett JE, Williamson PR (2014) Treatment options in invasive aspergillosis. Curr Treat Options Infect Dis 6: 309-325.

13. Labbé $\mathrm{A}-\mathrm{C}$, Su SH, Laverdière $M$, et al. (2007) High incidence of invasive aspergillosis associated with intestinal graft-versushost disease following nonmyeloablative transplantation. Biol Blood Marrow Transplant 13: 1192-1200.

14. Ljungman $P$, Bregni $M$, Brune $M$, et al. (2010) Allogeneic and autologous transplantation for haematological diseases, solid tumours and immune disorders: Current practice in Europe 2009. Bone Marrow Transplant 45: 219-234.

15. Tuncer HH, Rana N, Milani C, et al. (2012) Gastrointestinal and hepatic complications of hematopoietic stem cell transplantation. World J Gastroenterol 18: 1851-1860.

16. Garnett C, Apperley JF, Pavlů J (2013) Treatment and management of graft-versus-host disease: Improving response and survival. Ther Adv Hematol 4: 366-378.

17. Taccone FS, Van den Abeele AM, Bulpa P, et al. (2015) Epidemiology of invasive aspergillosis in critically ill patients: Clinical presentation, underlying conditions, and outcomes. Crit Care 19: 7.

18. Lamps LW, Lai KKT, Milner DA (2014) Fungal infections of the gastrointestinal tract in the immunocompromised host-An update. Adv Anat Pathol 21: 217-227.

19. Lee J-H, Lim G-Y, Im SA, et al. (2008) Gastrointestinal complications following hematopoietic stem cell transplantation in children. Korean J Radiol 9: 449-457.

20. Leonardelli F, Macedo D, Dudiuk C, et al. (2016) Aspergillus fumigatus intrinsic fluconazole resistance is due to the naturally occurring T301l substitution in Cyp51Ap. Antimicrob Agents Chemother 60: 5420-5426. 